\title{
Enfermedades registradas por contingencia laboral en descansos médicos emitidos en la Seguridad Social de Salud peruana 2015-2016
} Conditions reported as working occurrences in sick leave
certificates issued in Peruvian Social Security, 2015-2016

Correspondencia Erik Jair Jhonston Vela erik_honston@hotmail.com

Recibido: 08/05/2018 Arbitrado por pares Aprobado: 20/06/2018

Citar como: Jhonston EJ, OspinaSalinas E, Mendoza-Carrión A, Roncal-Ramirez RA, Bravo-Carrión $V$, Araujo-Castillo R. Enfermedades registradas por contingencia laboral en descansos médicos emitidos en la seguridad social de salud peruana 2015-2016. Acta Med Peru. 2018;35(2):116-20
Erik J. Jhonston 1,a, Estela E. Ospina-Salinas²,b,c, Alina M. Mendoza-Carrión ${ }^{2, d, e}$, R. Alexis Roncal-Ramírez ${ }^{2, f, g}$, Víctor M. Bravo-Carrión ${ }^{2, h, i}$, Roger Araujo-Castillo ${ }^{3, j}$

1 Unidad de Seguridad, Salud Ocupacional y del Medio Ambiente, Hospital Nacional Edgardo Rebagliati Martins. Lima, Perú.

2 Gerencia de Salud Ambiental, Seguridad y Salud en el Trabajo, EsSalud. Lima, Perú.

3 Instituto de Evaluación de Tecnologías en Salud e Investigación, EsSalud. Lima, Perú.

a Médico cirujano, b abogada, c especialista en Derecho del Trabajo y Seguridad Social, d médico psiquiatra, e magister en Salud Ocupacional y Medio Ambiente, f médico ocupacional, g magister en Salud Laboral, $\mathrm{h}$ ingeniero químico, i especialista en Gestión de riesgos y seguros en Seguridad y Salud en el Trabajo; j médico infectólogo.

\section{RESUMEN}

Objetivos: Describir las enfermedades registradas por contingencia laboral en los descansos médicos emitidos por el Seguro Social de Salud (EsSalud) a nivel nacional, 2015-2016. Materiales y métodos: Se realizó un estudio descriptivo transversal de la base total existente en EsSalud. El estudio abarcó todos los certificados de incapacidad temporal para el trabajo (CITT) reportados como enfermedades por contingencia laboral en el sistema de gestión hospitalario de EsSalud a nivel nacional. Resultados: De $256 \mathrm{CITT}$ reportados, ingresaron al análisis 188 CITT que cumplieron el criterio de inclusión (encontrarse en lista peruana de enfermedades profesionales). Los trastornos músculo esqueléticos representaron la patología más frecuente, presentando mayor cantidad de días por incapacidad. En 39 CITT no se logró identificar a que actividad productiva se encontraban registradas. Conclusiones: En las enfermedades registradas por contingencia laboral que requirieron descanso médico, la gran mayoría corresponde a trastornos musculo esqueléticos, dentro de las cuales el lumbago tuvo mayor frecuencia. Existe la necesidad de realizar mayor investigación para definir mediante los criterios de causalidad si las enfermedades mencionadas corresponden a la definición de enfermedades profesionales.

Palabras clave: Enfermedades profesionales; Seguro de salud; Omisiones de registro (fuente: DeCS BIREME). 


\begin{abstract}
Objectives: To describe the conditions reported as working occurrences (occupational diseases) in sick leave certificates issued in Peruvian Social Security (EsSalud) all over the country, for the 2015-2016 period. Materials and Methods: This is a descriptive cross-sectional study of the EsSalud database. The study comprised all sick leave certificates issued because of working occurrences in the EsSalud hospital management system all over the country. Results: Of 256 certificate reports, 188 that complied with the inclusion criterion (being included in the Peruvian list for occupational diseases) entered the analysis. Musculoskeletal conditions were the most frequently reported diseases, leading to the largest amount of days granted for sick leave. In 39 of the aforementioned certificates it was not possible to identify to which activity the condition was related to. Conclusions: Amongst conditions reported as working occurrences (occupational diseases) that required issuing a sick leave certificate, most of them were musculoskeletal conditions, and low back pain was the most prevalent. There is a need for performing further research in order to define according to causality criteria whether these conditions truly correspond to occupational diseases.
\end{abstract}

Keywords: Occupational diseases; Insurance, health; Underregistration (source: MeSH NLM).

\section{INTRODUCCIÓN}

La Organización Internacional del Trabajo (OIT) indica que una enfermedad es categorizada como laboral o profesional cuando ha sido contraída producto de la exposición a factores de riesgo inherentes al trabajo; además, ha estimado que se producen anualmente 160 millones de casos no mortales de enfermedades profesionales y 2,02 millones de casos mortales -5500 muertes diarias- ${ }^{[1]}$.

En los Estados Unidos se reportaron aproximadamente 862200 casos de enfermedades profesionales y 60300 muertes al año ${ }^{[2]}$; en Canadá, entre 77900 a 112000 casos y entre de 2381 y 6010 muertes por año ${ }^{[3]}$; en España, 22000 casos anuales -cifra que ascendería hasta los 88000 casos- y 13000 muertes anuales ${ }^{[4,5]}$; en Argentina, durante el año 2016, se reportaron 16993 casos ${ }^{[6]}$; en Chile, el reporte anual promedio es de 2556 casos $^{[7]}$; en Colombia, para el año 2000, se estimaron 101645 casos $^{[8]}$. En nuestro país, el Ministerio del Trabajo y Promoción del Empleo (MTPE) solo notificó 93 casos de enfermedades profesionales en el 2015, y 32 en el $2016^{[9]}$.

Las enfermedades profesionales y los accidentes laborales significan un costo social considerable pues representan una pérdida anual del $4 \%$ del Producto Bruto Interno en el mundo ${ }^{[10]}$, porcentaje que llegaría a un $10 \%$ en América Latina ${ }^{[11]}$. Por lo tanto, consideramos necesario que se describan las características de las enfermedades registradas por contingencia laboral, información que nos permitirá concluir si es que la definición de enfermedad profesional es adecuadamente utilizada, ya que esta problemática suele estudiarse solo en función de la magnitud -variable común en estudios de este tipo- dejando de lado la causalidad. Este incorrecto enfoque podría provocar el subregistro de casos, lo que disminuiría el conocimiento de su magnitud e impacto económico. El presente estudio busca describir las enfermedades registradas por contingencia laboral que generaron descansos médicos en el Seguro Social de Salud del Peru (Essalud).

\section{MATERIALES Y MÉTODOS}

Se realizó un estudio descriptivo transversal. Se incluyó a todos los certificados de incapacidad temporal para el trabajo (CITT) que describían como contingencia a una enfermedad profesional, reportados en el sistema de gestión hospitalario de EsSalud a nivel nacional. El periodo estudiado correspondió a los años 2015 y 2016. Se consideró como enfermedad profesional a aquella patología cuyo código CIE-10 (Clasificación Internacional de Enfermedades, $10^{\circ}$ edición) se encontrara dentro de la lista peruana de enfermedades profesionales ${ }^{[12]}$.

La información recolectada proviene de trabajadores asegurados en EsSalud que requirieron días de descanso médico por enfermedades de origen laboral, diagnosticadas por el médico que prestó la atención.

Tabla 1. Distribución de las características de los CITT por grupo etario, sexo y año de emisión.
Variable

\section{Grupo (años) \\ $\leq 20$}

$21-30$

$31-40$

$41-50$

$51-60$

$>60$

\section{Sexo}

Masculino

$104(55,32)$

Femenino

$84(44,68)$

Año

2015

$32(17,02)$

2016

$156(82,98)$

$\mathbf{N}^{\circ}$ de días de incapacidad

5564
CITT: certificado de incapacidad temporal para el trabajo 
Los CITT contienen indispensablemente el tipo de contingencia (enfermedad, accidente, etc.) y el periodo de incapacidad temporal para el trabajo. Son documentos médicos legales que, además, incluyen información sobre edad, sexo, diagnóstico según código CIE-10, número de días de descanso médico, registro único de contribuyentes (RUC) de la empresa empleadora, entre otros.

La identificación de la actividad productiva de la empresa empleadora se obtuvo a través de la página "Consulta RUC" (https://e-consultaruc.sunat.gob.pe/cl-ti-itmrconsruc/jcrS00Alias) de la Superintendencia Nacional de Aduanas y Administración Tributaria (SUNAT). Obtuvimos el código de actividad productiva mediante el ingreso del número de RUC de la empresa. El código usado para esta categorización corresponde a la Clasificación Internacional Industrial Uniforme (CIIU).

Al recabar la información de la base de datos de EsSalud, nos vimos limitados de obtener solo los datos que ésta registraba, por lo que no pudimos recuperar información importante como lugar en donde se emitió el CITT, profesión del trabajador o establecimiento que emitió el CITT.

Se elaboró una base de datos en una hoja de cálculo Microsoft Excel ${ }^{\circledR}$, se eliminó la información relacionada a la identidad y luego se analizó en Epi Info 7,2 (CDC, Atlanta, GA). El análisis descriptivo consistió en frecuencias, porcentajes y medianas.

El estudio fue revisado y aprobado por el Comité de Investigación y ética del Hospital Nacional Edgardo Rebagliati Martins para su desarrollo y publicación.

\section{RESULTADOS}

Del total de 256 CITT identificados para los años 2015 y 2016, se excluyeron 68 por tener un código $\mathrm{CIE}-10$ no aceptado dentro de la normativa peruana ${ }^{[12]}$. Al final, incluimos en nuestro análisis a $188 \mathrm{CITT}(73,4 \%)$.

La distribución de la edad, y los días de incapacidad para el trabajo no presentaron una distribución normal (Shapiro Wilk < 0,01), la mediana de edad fue 39 años (rango intercuartil: 33 51 años), la distribución por grupos etarios, sexo y por años se muestra en la Tabla 1, la mediana para días de descanso medico fue de 6 días (rango intercuartil: 3-30 días). Se encontró que los trastornos músculo esqueléticos (TME) representaron la patología más frecuente, así mismo también fueron los que más días de incapacidad generaron (Tabla 2). Los diagnósticos CIE-10 más frecuentes en el grupo de enfermedades profesionales, fueron los lumbagos $(28,7 \%)$, seguido por lesiones del manguito del rotador $(5,9 \%)$, y en tercer lugar la tuberculosis pulmonar (5,9\%). Al observar los días acumulados por patologías, el síndrome del túnel del carpo fue el que más días de incapacidad presento $(11,7 \%)$, seguido de dedos en gatillo $(9,7 \%)$ y en tercer lugar la tuberculosis pulmonar (9,5\%) (Tabla 3).

Al evaluar las actividades económicas de las empresas empleadoras observó que 39 de los CITT no registraron el RUC, imposibilitando definir a que actividad productiva se encuentran inscritas, luego en frecuencia se encontró que las empresas
Tabla 2. Distribución de los CITT por grupo de enfermedades.

\begin{tabular}{lccc} 
Grupo de enfermedad & $\begin{array}{c}\mathbf{N}^{\circ} \text { de CITT } \\
\text { (n=188) }\end{array}$ & $\begin{array}{c}\mathbf{N}^{\circ} \text { de } \\
\text { días de } \\
\text { incapacidad } \\
\text { (n=5 564) }\end{array}$ & $\begin{array}{c}\text { Me } \\
\text { (RIC) }\end{array}$ \\
\hline $\begin{array}{l}\text { Trastorno musculo } \\
\text { esquelético }\end{array}$ & 147 & 4021 & $5(3-8)$ \\
$\begin{array}{l}\text { Trastorno del sistema } \\
\text { respiratorio }\end{array}$ & 26 & 1078 & $30(10-58)$ \\
$\begin{array}{l}\text { Trastornos de la piel } \\
\text { Trastornos neurológicos }\end{array}$ & 5 & 116 & $5(3-8)$ \\
Trastornos del ojo & 3 & 338 & $12(8-30)$ \\
\hline
\end{tabular}

CITT: certificado de incapacidad temporal para el trabajo; Me: mediana; RIC: rango intercuartílico.

catalogadas dentro de las "actividades de administración pública en general", en igual frecuencia con "Otras actividades manufactureras" (Tabla 4).

\section{DISCUSIÓN}

Reconocemos que nuestro estudio, por sus características, presenta importantes limitaciones y sesgos propios de usar una fuente secundaria; la base utilizada tiene sesgos de información lo que no permitió realizar un análisis específico sobre enfermedades por contingencia laboral. A pesar de estas limitaciones, se pone en evidencia que en nuestro país existe dificultad en el registro y notificación de las enfermedades profesionales, esto es aún más evidente al comparar la notificación de casos de países vecinos, lo que plantea la necesidad de investigar esta brecha referente a la notificación de los casos.

En nuestro país, son las Instituciones Prestadoras de Servicios de Salud (IPRESS) del sector público, privado, militares, policiales o de la seguridad social las responsables de notificar las enfermedades profesionales, en donde los profesionales médicos no necesariamente ocupacionales, certifican la contingencia laboral o común ${ }^{[13]}$.

Dentro del sistema de salud peruano existe la posibilidad que enfermedades por contingencia laboral inadecuadamente consideradas como enfermedad común estén siendo atendidas por el seguro regular de EsSalud, el cual no tiene cobertura para contingencias laborales ${ }^{[14]}$; lo que generaría un inadecuado uso de los recursos. Si dichas enfermedades por contingencia laboral, mediante una investigación, se definieran como enfermedad profesional deberían ser cubiertas por el Seguro complementario de trabajo de Riesgo ${ }^{[15,16]}$. Los autores sugerimos la creación de consultorios especializados de medicina ocupacional en las IPRESS, que den soporte a los médicos asistenciales, con el objetivo de determinar la causalidad y definir si son enfermedades profesionales aquellas enfermedades consideradas como contingencia laboral y aportando en la notificación de los mismos.

El sexo masculino resultó más afectado, proporción que puede variar según la población estudiada, algo que sucede en otros estudios. Sobre el grupo etario más afectado se observó que 
Tabla 3. Distribución de patologías más frecuentes* de los CITT.

\begin{tabular}{|c|c|c|c|}
\hline Enfermedad & $\begin{array}{c}N^{\circ} \text { de CITT } \\
(n=188)\end{array}$ & $\begin{array}{c}\mathrm{N}^{\circ} \text { de días de } \\
\text { incapacidad } \\
(\mathrm{n}=5 \mathrm{564)}\end{array}$ & $\begin{array}{l}\text { Me } \\
\text { (RIC) }\end{array}$ \\
\hline Lumbago no especificado & 37 & 160 & $3(2-4)$ \\
\hline Lumbago con ciática & 17 & 125 & $6(2-11)$ \\
\hline Síndrome del manguito rotatorio & 11 & 343 & $15(4-56)$ \\
\hline Tuberculosis pulmonar & 11 & 529 & $30(30-60)$ \\
\hline Síndrome de túnel de carpo & 9 & 654 & $38(7-80)$ \\
\hline Tenosinovitis de estiloides radial & 7 & 144 & $7(3-22)$ \\
\hline Trastornos de disco lumbar y otros, con radiculopatía & 7 & 207 & $15(6-36)$ \\
\hline Dedo en gatillo & 5 & 544 & $82(4-174)$ \\
\hline Otras sinovitis y tenosinovitis & 5 & 59 & $6(4-7)$ \\
\hline $\begin{array}{l}\text { Trastornos de los tejidos blandos relacionados con el uso, el uso } \\
\text { excesivo y presión }\end{array}$ & 5 & 462 & $115(34-133)$ \\
\hline Trastornos de plexo braquial & 4 & 508 & $150(87-167)$ \\
\hline
\end{tabular}

* Solo se detallan las 11 patologías más frecuentes en función del número de CITT.

CITT: certificado de incapacidad temporal para el trabajo; Me: mediana; RIC: rango intercuartílico.

Tabla 4. Distribución de los CITT por las principales actividades productivas *.

\begin{tabular}{lccc}
\multicolumn{1}{c}{ Actividades productivas } & $\begin{array}{c}\mathbf{N}^{\circ} \text { de CITT } \\
\text { (n=188) }\end{array}$ & $\begin{array}{c}\mathbf{N}^{\circ} \text { de días de } \\
\text { incapacidad } \\
\text { (n=5 564) }\end{array}$ & $\begin{array}{c}\text { Me } \\
\text { (RIC) }\end{array}$ \\
No se registró actividad & 39 & 1018 & $11(6-30)$ \\
Actividad administrativa publica en general & 13 & 89 & $3(2-30)$ \\
Otras industrias manufactureras & 13 & 904 & $5(2-30)$ \\
Servicios públicos de seguridad social & 10 & 361 & $12,5(6-60)$ \\
Actividades de hospitales & 8 & 133 & $16(4-30)$ \\
Fabricación de jabones y detergentes & 7 & 515 & $5(4-16)$ \\
Extracción de minerales metalíferos no ferrosos & 6 & 311 & $3(2-23)$ \\
Construcción de edificios completos & 5 & 324 & $5(3-30)$ \\
Actividades limpieza de edificios & 4 & 28 & $4(2-7)$ \\
Fabricación de artículos de cuchillería y ferretería & 4 & 407 & $99,5(75-129)$ \\
\hline
\end{tabular}

* Solo se detallan las 11 patologías más frecuentes en función del número de CITT.

CITT: certificado de incapacidad temporal para el trabajo; Me: mediana; RIC: rango intercuartílico.

casi la mitad de los casos se concentran entre la edad de 31 y 50 años (ver Tabla 1); esta situación ocurriría debido a la existencia de ciertas enfermedades profesionales que requieren un largo tiempo de exposición a noxas para se puedan presentar (enfermedades con largo periodos prepatogénicos, como por ejemplo la silicosis) ${ }^{[4,5]}$. Al comparar los casos entre el año 2015 y 2016, se observó una diferencia entre años; este aumento podría ser consecuencia del aumento en el mal reporte, o por un aumento real de casos, el cual amerita una futura investigación, pero es necesario mencionar que en EsSalud no se instaló medidas para mejorar el reporte de descansos médicos relacionados a enfermedades por contingencia laboral.

Dentro del grupo de enfermedades más prevalentes, los TME representaron el $78,2 \%$ de las enfermedades por contingencia laboral más frecuente, seguido por enfermedades respiratorias $(13,8 \%)$ concordando en proporción similar con los días perdidos por incapacidad temporal que en total sumaron 5564 días entre los dos años (aproximadamente 15 años en CITT). Esta información representa un sesgo, ya que existen patologías de origen ocupacional que no generan descansos médicos (por ejemplo: hipoacusias y dermatitis), hecho respaldado por estudios en las cuales los TME, junto con las patologías del oído (hipoacusia inducida por ruido), estrés, ansiedad son las más prevalentes ${ }^{[4,5]}$.

Al desglosar los principales TME, los lumbagos representan la patología más prevalente, seguida por el síndrome de manguito de los rotadores, sin embargo al observar los días de incapacidad temporal, el síndrome del túnel del carpo, es el que más días acumula seguido por la tuberculosis pulmonar, la cual no 
podemos definir su origen ocupacional, pues según la Dirección General de Epidemiologia del Ministerio de Salud (MINSA) los grupos ocupacionales más afectados entre el 2013-2014 fueron oficiales y operarios de construcción $(8,1 \%)$, los trabajadores de los servicios, vendedores de comercios y mercados (7\%), agricultores y trabajadores calificados agropecuarios, forestales y pesqueros $(6,3 \%)$ y los profesionales y técnicos de Salud con un $1,6 \%$ del total ${ }^{[17]}$.

Además, también se describió las actividades económicas a las cuales las empresas. Es preciso enfatizar que 39 de los CITT no registraron el RUC de empresa, lo que impidió definir la actividad económica a la que pertenecían; seguida en frecuencia por las actividades económicas definidas como "Administración pública en general" y "otras industrias manufactureras". Esta frecuencia puede ser resultado de la distribución de la población económicamente activa por sector económico, estimada por la OMS para Latinoamérica, sin embargo según las estadísticas nacionales reportadas por el Instituto Nacional de Estadística e informática, las principales actividades productivas para nuestro país en el 2016 fueron agricultura, manufactura y construcción, donde se concentró gran parte de la población económicamente activa, y donde se podría esperar más casos de enfermedad por contingencia laboral ${ }^{[18,19]}$. En España, por ejemplo, las industrias manufactureras fueron las que más reportaron enfermedades profesionales, en Perú aún no contamos con este tipo de estadísticas ${ }^{[4,5]}$. Es importante mencionar que el CIIU que registran muchas veces las empresas no refleja exactamente a la actividad económica que ejercen, representando probablemente una inadecuada aproximación de la realidad.

A pesar de todas las limitaciones mencionadas, los resultados nos hacen sugerir que se realice una mayor investigación sobre enfermedades profesionales, y que se creen mecanismos que permitan contar con consultorios especializados de medicina ocupacional para investigación de los criterios de causalidad, que permitan diferenciar la enfermedad común de la profesional, así como reforzar el procedimiento de notificación en la IPRESS, y simplificar la notificación de casos al MTPE.

Fuente de financiamiento: el presente trabajo fue autofinanciado. Conflicto de interés: los autores manifestamos no tener conflictos de interés.

\section{REFERENCIAS BIBLIOGRÁFICAS}

1. Organización Internacional del Trabajo. XIX Congreso sobre Seguridad y Salud en el Trabajo [Internet]. 11-15 de setiembre de 2011. Estambul, Turquía: Organización Internacional del Trabajo, Asociación Internacional de la Seguridad Social, Ministerio de Trabajo y Seguridad Social de la República de Turquía; 2011 [citado 4 de noviembre de 2017]. Disponible en: http://www.lo.org/global/ about-the-ilo/newsroom/news/WCMS_162661/lang--es/index.htm

2. Leigh JP, Markowitz SB, Fahs M, Shin C, Landrigan PJ. Occupational injury and illness in the United States. Estimates of costs, morbidity, and mortality. Arch Intern Med. 1997;157(14):1557-68.
3. Kraut A. Estimates of the extent of morbidity and mortality due to occupational diseases in Canada. Am J Ind Med. 1994;25(2):267-78.

4. García García AM, Gadea Meríno R, López Martínez V. Estimación de la mortalidad atribuible a enfermedades laborales en España, 2004. Rev Esp Salud Pública. 2007;81(3):261-70.

5. García AM, Gadea R. Estimaciones de incidencia y prevalencia de enfermedades de origen laboral en España. Aten Primaria. 2008;40(9):439-45.

6. Organización Internacional del Trabajo. La Prevención de las enfermedades profesionales [Internet]. Ginebra: OIT; 2013 [citado 5 de noviembre de 2017]. Disponible en: http://www.ilo.org/ safework/info/publications/WCMS_209555/lang--es/index.htm

7. García A. Análisis de las Enfermedades Profesionales en la Asociación Chilena de Seguridad (ACHS): Descripción de la Situación en el Periodo 1995-2009. Cienc Trab. 2011;13(40):107-12.

8. Idrovo AJ. Estimation of the incidence of occupational diseases in Colombia, 1985-2000. Rev Salud Pública. 2003;5(3):263-71.

9. Perú, Ministerio de Trabajo y Promoción del Empleo. Anuarios Estadísticos [Internet]. Lima: MTPE; 2017 [citado 5 de noviembre de 2017]. Disponible en: http://www2.trabajo.gob.pe/estadisticas/ anuarios-estadisticos/

10. Organización Internacional del Trabajo. Comunicado conjunto OIT/OMS: El número de accidentes y enfermedades relacionados con el trabajo sigue aumentando OIT y OMS recomiendan aplicar estrategias de prevención [Internet]. Ginebra: OIT; 2005 [citado 5 de noviembre de 2017]. Disponible en: http://www.ilo.org/global/ about-the-ilo/newsroom/news/WCMS_006102/lang--es/index.htm

11. Fontes lunes R. Seguridad y salud en el trabajo en América latina y el caribe: Análisis, temas y recomendaciones de política [Internet]. Banco Interamericano de Desarrollo, 2002 [citado 5 de noviembre de 2017]. Disponible en: https://publications.iadb. org/bitstream/handle/11319/5260/Seguridad\%20y\%20salud\%20 en\%20el\%20trabajo\%20en\%20Am\%C3\%A9rica\%20Latina\%20 y\%20el\%20Caribe\%3A\%20an\%C3\%A1lisis\%2C\%20temas\%20y\%20 recomendaciones\%20de\%20pol\%C3\%ADtica.pdf?sequence $=1$

12. Norma Técnica de Salud que establece el Listado de Enfermedades Profesionales. Diario Oficial El Peruano. Resolución Ministerial No 480-2008/MINSA (14 julio del 2008).

13. Reglamento de Seguridad y Salud en el Trabajo. Diario Oficial El Peruano. Decreto Supremo No 009-2005-TR (setiembre del 2008).

14. Ley de Modernización del Seguro Social en Salud. Diario Oficial El Peruano. Ley 26790 (17 mayo de 1997).

15. Reglamento de la Ley de Modernización de La Seguridad Social en Salud. Diario Oficial El Peruano. Decreto Supremo No 009-97-SA (setiembre de 1997).

16. Normas Técnicas del Seguro Complementario de Trabajo de Riesgo. Diario Oficial El Peruano. Decreto Supremo Nº03-98-SA (abril de 1998).

17. Ministerio de Salud del Perú. Análisis de la situación epidemiológica de la tuberculosis en el Perú, 2015 [Internet]. Lima: Minsa; 2016 [citado 6 de noviembre de 2017]. Disponible en: http://www.dge.gob.pe/ portal/index.php?option=com_content\&view=article\&id=599:asistbc-2015\&catid=20:institucional\&ltemid=204

18. Nelson DI, Concha-Barrientos $M$, Driscoll T, Steenland $K$, Fingerhut $M$, Punnett $L$, et al. The global burden of selected occupational diseases and injury risks: Methodology and summary. Am J Ind Med. 2005;48(6):400-18.

19. Instituto Nacional de Estadística e Informática. Población económicamente activa ocupada [Internet]. Lima: INEI [citado 15 de julio de 2018]. Disponible en: https://www.inei.gob.pe/estadisticas/ indice-tematico/economia/ 